\title{
An assessment of the dynamic interaction of the rolling stock and the long-span bridges on high-speed railways
}

\author{
Leonid K. Dyachenko ${ }^{1, *}$ and Andrey V. Benin ${ }^{1}$ \\ ${ }^{1}$ Emperor Alexander I St. Petersburg State Transport University, 190031Moskovsky pr. 9, St. \\ Petersburg, Russia
}

\begin{abstract}
When the high-speed railway traffic is being organized, it becomes necessary to elaborate bridge design standards for high-speed railways. To ensure safety of traffic and reliability of bridges one needs to resolve the dynamic problems of various types of high-speed trains moving along the structures. The article analyzes the magnitude of inertial response as the function of the external stress parameters. A simplified structure assessment technique is offered based on the criterion of passenger comfort while crossing the bridges. The usefulness of the given research arises from the reduction of complexity of the complicated dynamic calculations needed to describe a high-speed train travelling along the artificial structures. Dependences of relative elastic deflection values from the span length are proposed, which allows designing and calculating long-span structures using the simplified dynamic models.
\end{abstract}

\section{Introduction}

One of the main peculiarities of bridge design on high-speed railways (HSR) is an obligatory requirement to account for the dynamic interaction of the high-speed rolling stock and the bridge structure. Meanwhile, unlike it is provided by the existing Russian standards, where the dynamic interactions in the 'bridge-train' system are calculated using a system of coefficients (a quasistatic model), at high-speed traffic the dynamic interaction factor should be accounted for by performing a dynamic task [1, 2, 3]. Specificity of the rolling stock power load on the bridge structure is due to the so called speed effect of kinematic excitation, which means that the temporary power action caused by the bridge live load is transferred to the bridge superstructure via the wheel sets of the rolling stock. The most evident power action is posed on the bridge by trains consisting of single-type cars $[2,3]^{*}$

While operating a HSR bridge, the speed effect can cause a condition of resonance and lead to dangerous vibrations of the bridge structures. This occurs in case the period of train power action coincides with the main period of the bridge superstructure self-induced vertical vibrations [3]. The dynamic effects not only result in the increase of stress within

*Corresponding author: leonid_dyachenko@mail.ru 
the bridge elements, but also they exert an unfavourable influence on the stability of the bridge deck and passenger comfort.

\section{Task assignment and design model selection}

A dynamic task of the 'bridge-train' system interaction is solved mainly by means of computational simulation. Selection of a design model, thus, depends on the task assigned and - as a consequence - on the necessary specification of the 'bridge-train' system elements. It is possible to use various dynamic models [3]:

I. 'Moving powers on a beam' type. The superstructure is simulated with an elastic beam of finite mass and viscous damping, whereas the train is simulated with a system of moving powers (Fig. 1). Such a model allows to account for the speed effect and to obtain the critical speed values as well as peak values of the superstructure deformations and accelerations.

II. 'Moving masses on a beam' type. The superstructure is simulated similar to the model I, whereas the train is simulated with a system of moving masses with elastic and viscous restraints (Fig. 2). This approach allows to assess the extent of passenger comfort on the basis of acceleration analysis in the car bodies as well as the impact of track and wheel defects on the magnitude of the power of the rolling stock running gear dynamic interaction.

Calculations show that for spans no longer that $50 \mathrm{~m}$ the main criterion determining the 'bridge-train' system interaction are the resonance phenomena. The tasks of defining the resonance modes of vibration, critical speeds, dynamic coefficients are evidentially formulated by using Model I. At the same time, when designing long-span bridges according to the Russian and European norms, one should solve a dynamic problem of the 'bridge-train' interaction using Model II [4, 5]. This research comprises an analysis of distinctive problems of the 'bridge-train' system interaction for long-span bridges and is designated to define the main criteria and recommendations to assign the vertical elasticlimit deformations caused by rolling stock as a criterion of providing passenger comfort.

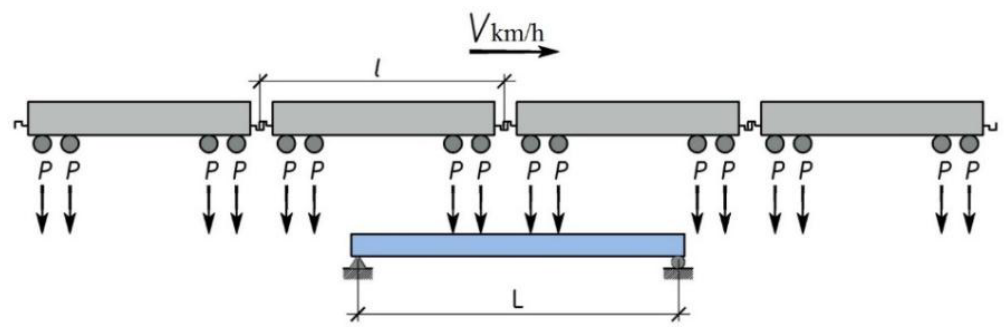

Fig. 1. 'Moving Powers on a Beam' Model for Dynamic Calculations.

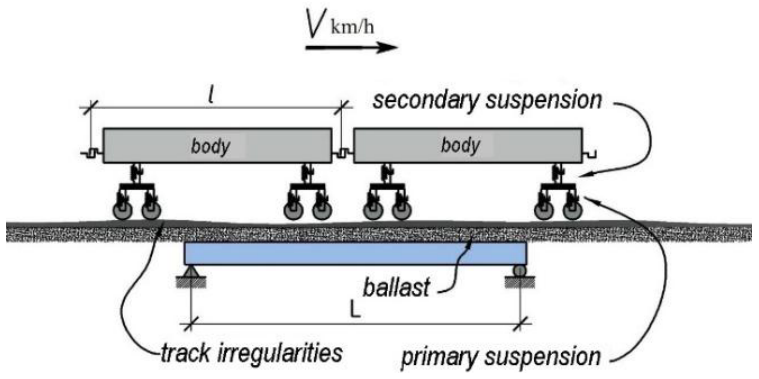

Fig. 2. 'Moving Masses on a Beam' Model for Dynamic Calculations. 
The criterion of passenger comfort while travelling along a bridge is ensured by limiting the superstructure elastic deflections, which is a kind of irregularity in the path of motion (kinematic excitation) causing the cars to vibrate. In this case, the vibrations and corresponding car accelerations depend on:

- the car dynamic parameters (rigidity and damping properties of the primary and secondary suspension, distribution of sprung and unsprung masses, car geometry, etc.);

- the magnitude of the superstructure elastic deflection (magnitude of irregularity);

- the magnitude of camber and initial track up-grade.

The main parameter characterizing the dynamic 'car' system is its natural frequency according to the first vertical form (chattering). It is known that the frequency of HSR car vertical vibrations ranges from 0.8 to $1.5 \mathrm{~Hz}$ depending on the type of train. Let us introduce a notion of the car resonance speed that is equal to the product of span length multiplied by the car vibration frequency. In other words, the speed at which the kinematic excitation has a frequency equal to the natural car frequency:

$$
V_{c a r}^{c r t}=v_{c a r} \cdot L,
$$

where

$V_{c a r}^{c r t}$ - car resonance speed at a bridge;

$v_{\text {car }}$ - natural car frequency;

$L-$ span length.

Taking into account the range of possible HSR train types, let us consider the frequencies from 0.8 to $1.5 \mathrm{~Hz}$ and define the span lengths which will undergo car resonance vibrations (Table 1).

Table 1. Span lengths corresponding with the car resonance vibrations for various speeds.

\begin{tabular}{|c|c|c|c|c|}
\hline \multirow{2}{*}{$\mathbf{V}[\mathbf{k m} / \mathbf{h}]$} & \multicolumn{4}{|c|}{ Car Vibration Frequency [Hz] } \\
\cline { 2 - 5 } & $\mathbf{0 . 8}$ & $\mathbf{1}$ & $\mathbf{1 . 2 1 5}$ & $\mathbf{1 . 5}$ \\
\hline $\mathbf{2 0 0}$ & 69.4 & 55.6 & 45.7 & 37.0 \\
\hline $\mathbf{2 5 0}$ & 86.8 & 69.4 & 57.2 & 46.3 \\
\hline $\mathbf{3 0 0}$ & 104.2 & 83.3 & 68.6 & 55.6 \\
\hline $\mathbf{3 5 0}$ & 121.5 & 97.2 & 80.0 & 64.8 \\
\hline
\end{tabular}

The path of train's motion is also of critical importance. Various proportions of span and car lengths cause the path's form to change. Let us suppose that in case the span length $L$ is more than twice as much as the car length, the line of the superstructure elastic deflection will coincide with the sinusoid half-wave and - due to little irregularity of the rolling stock axles load - stay almost unchangeable in time when a train is moving along the structure. At the given proportion of the span and the car lengths resonance phenomena do not arise. Nevertheless, on short and medium spans resonance vibrations of the superstructure might occur when the bridge natural frequency coincides with the frequency of the external action. When resonance vibrations occur, the train path of motion will change according to the superstructure deflection pulsation. It should be noted that this condition not only causes car vibration but also - what is especially important - results in minute local wheel unloading during the contact with rail.

This paper considers more than $50 \mathrm{~m}$ long spans and proposes calculations of the 'bridge-train' system dynamic interaction for Velaro RUS ("Sapsan") high-speed train 
passing along bridge structures. The modal analysis conducted showed that the car vibration frequency according to the vertical form (chattering) is $1.215 \mathrm{~Hz}$.

As a structure model we have selected a chain of superstructures with a single span length, which had been changing in the course of calculation. In the resonance mode of car vibrations the number of spans in a chain plays an important role or - in other words duration of kinematic action applied to the rolling stock. However, on chains of 10 or more spans vibrations of cars moving along the structure become stable. For structures with shorter spans the adjustment reduction factors are showed in Table 2. It is also interesting how the maximum accelerations in car bodies are distributed according to their position within the train (Fig. 3).

Table 2. Adjustment reduction factors for car body accelerations according to the number of spans.

\begin{tabular}{|l|c|c|c|c|c|c|}
\hline \multirow{3}{*}{ Adjustment factor } & \multicolumn{6}{|c|}{ Number of spans } \\
\cline { 2 - 8 } & $\mathbf{1}$ & $\mathbf{2}$ & $\mathbf{3}$ & $\mathbf{5}$ & $\mathbf{7}$ & $\mathbf{1 0}$ \\
\cline { 2 - 8 } & 0.40 & 0.62 & 0.74 & 0.91 & 0.98 & 1.00 \\
\hline
\end{tabular}

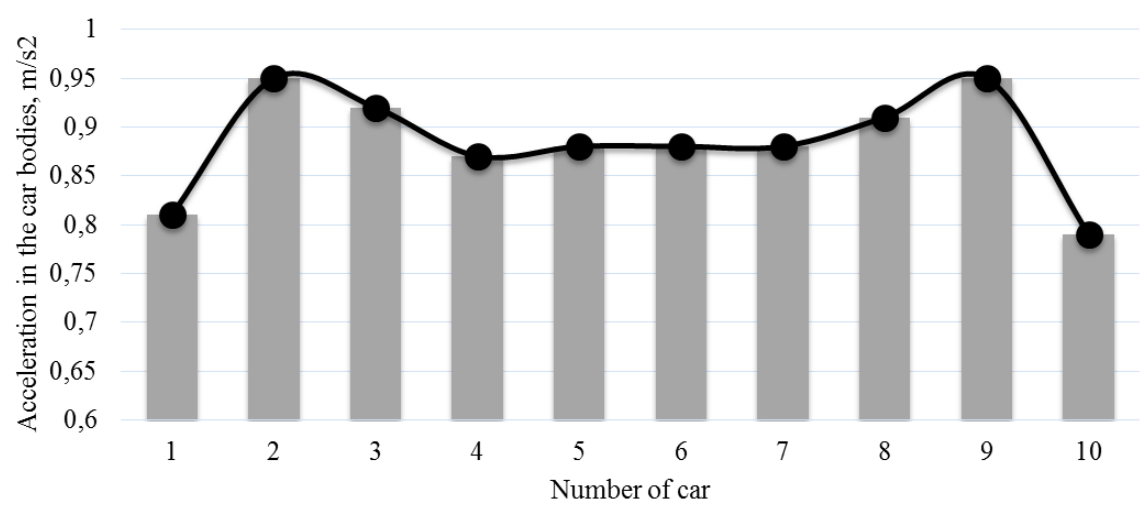

Fig. 3. Distribution of maximum acceleration in the car bodies according to their position within the train moving along the bridge structures.

\section{Limitations for vertical elastic deflections}

Basing on the models described we have obtained the dependency relations and showed a graph of vertical elastic deflections limitation according to the train speed and span length (Fig. 4). A serious difference from the European norms [5] is the fact that the graph is not limited by the span length of $100 \mathrm{~m}$, which allows to estimate passenger comfort on the long span without making time-consuming dynamic calculations by Model II. Moreover, the graph accounts for the temporary load CK $(K=8)$ adopted in Russia rather that for the European LM71 load, which can be seen as a kind of norms harmonization. Table 3 comprises parameters of the relative deflections $(\delta)$ standardization for various traffic speeds. 


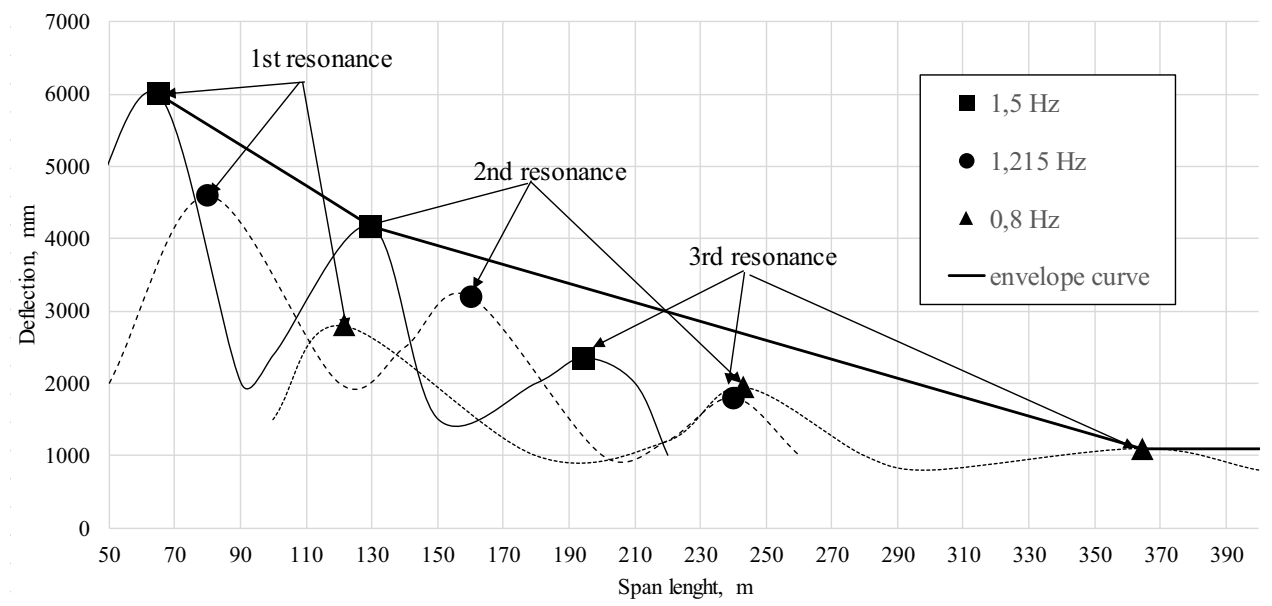

Fig. 4. Limitation of the superstructure vertical deflections aimed to provide maximum accelerations in car bodies of $1.0 \mathrm{~m} / \mathrm{s}^{2}$ for train speed up to $350 \mathrm{~km} / \mathrm{h}$.

Table 3. Parameters of the relative deflections standardization for various traffic speeds

\begin{tabular}{|c|c|c|c|c|c|c|}
\hline $\begin{array}{c}\mathbf{2 5 0} \\
{[\mathbf{k m} / \mathbf{h}]}\end{array}$ & $\mathbf{4 6}$ & $\mathbf{9 3}$ & $\mathbf{1 4 0}$ & $\mathbf{1 7 2}$ & $\mathbf{4 0 0}$ & $\boldsymbol{L}[\mathbf{m}]$ \\
\cline { 2 - 7 } & 3920.0 & 1650.0 & 1100.0 & 800.0 & 800.0 & $\boldsymbol{L} / \boldsymbol{\delta}$ \\
\hline $\begin{array}{c}\mathbf{3 0 0} \\
{[\mathbf{k m} / \mathbf{h}]}\end{array}$ & $\mathbf{5 6}$ & $\mathbf{1 1 0}$ & $\mathbf{3 1 2}$ & $\mathbf{4 0 0}$ & $\boldsymbol{L}[\mathbf{m}]$ \\
\cline { 2 - 7 } & 5100.0 & 2870.0 & 800.0 & 1100 & $\boldsymbol{L} / \boldsymbol{\delta}$ \\
\hline $\begin{array}{c}\mathbf{3 5 0} \\
{[\mathbf{k m} / \mathbf{h}]}\end{array}$ & $\mathbf{6 5}$ & $\mathbf{1 3 0}$ & $\mathbf{3 6 5}$ & $\mathbf{4 0 0}$ & $\boldsymbol{L}[\mathbf{m}]$ \\
\cline { 2 - 7 } & 6000.0 & 4175.0 & 1100.0 & 1100 & $\boldsymbol{L} / \boldsymbol{\delta}$ \\
\hline
\end{tabular}

As an example of applicability of the presented passenger comfort assessment method we have performed a calculation for a frame-and-beam-type reinforced concrete bridge with $110+110 \mathrm{~m}$ spans.

According to the norms $[4,5]$, when defining the admissible elastic deflections, the design length of continuous and frame-type superstructures shall be calculated as a straight average of span lengths multiplied by 1.2 coefficient. Thus, for a frame superstructure the design length is $132 \mathrm{~m}$. At the graph (Fig. 4) one can see that this span corresponds with the second car resonance with $1.5 \mathrm{~Hz}$ frequency; that is why it is this train that was taken for calculation. Basing on the same graph we have determined the maximum admissible relative deflection caused by live load C8 [4] and the minimum vertical rigidity of the superstructure. The adjustment reduction coefficient for a two-span structure (equal to 0.62) was also accounted for. Hence, the admissible deflection caused by the load C8 equals 51 mm.

Below one can find the accelerograms of the maximum vertical accelerations (Fig. 5) in the design train cars (natural frequency $-1.5 \mathrm{~Hz}$ ) moving along a reinforced concrete frame-type superstructure with $110+110 \mathrm{~m}$ spans at a speed of $350 \mathrm{~km} / \mathrm{h}$, which corresponds with the second resonance car speed. 
a)

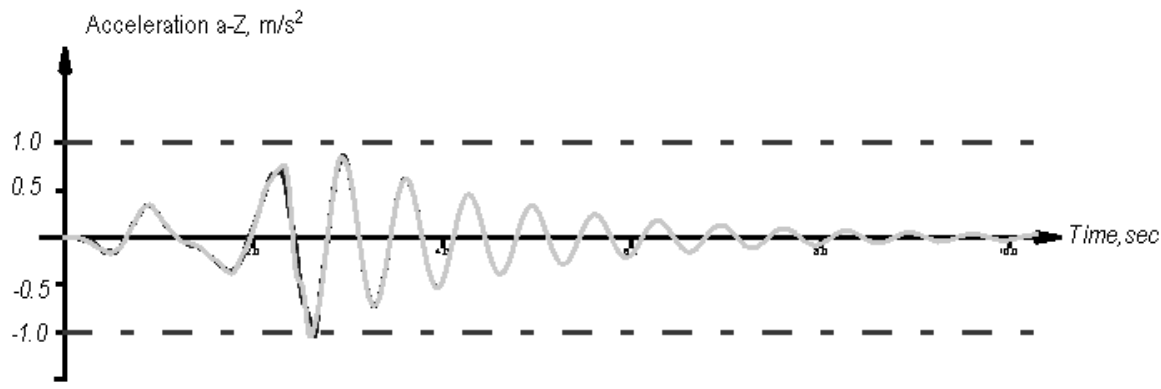

b)

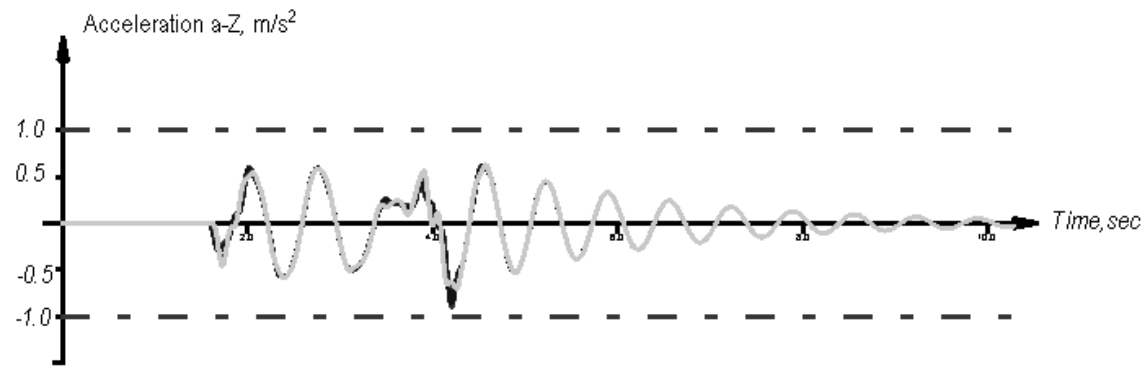

c)

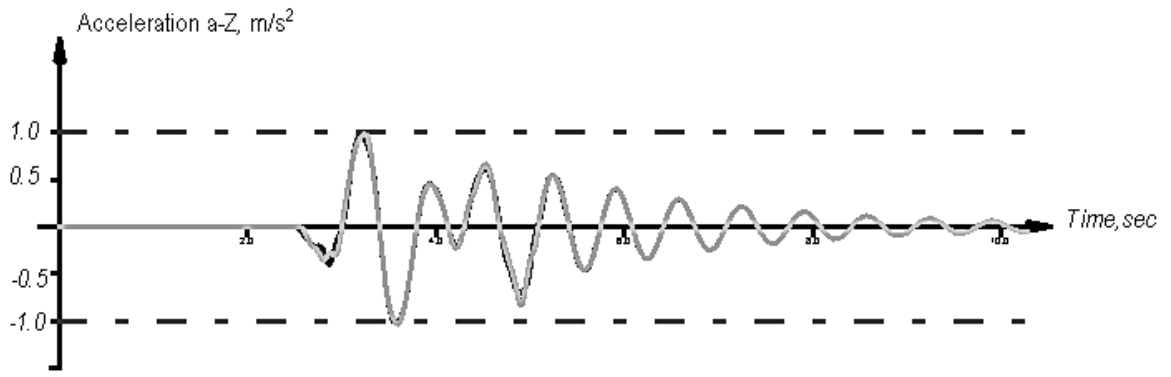

Fig. 5. Vertical accelerations in the design train cars moving along a frame-type superstructure with $110+110 \mathrm{~m}$ span at a speed of $350 \mathrm{~km} / \mathrm{h}$ : a - car №2; b - car №6; c - car №9.

\section{Conclusion}

To ensure safety of high-speed traffic and reliability of bridges during the whole lifetime one needs to resolve the dynamic problems of various types of high-speed trains moving along the structures. The article analyzes the dependences of the 'bridge-train' system interaction from the parameters of external stress. A simplified structure vertical rigidity assessment technique is offered based on the criterion of passenger comfort while crossing the bridges. The usefulness of the given research arises from the reduction of complexity of the complicated dynamic calculations needed to describe a high-speed train travelling along the artificial structures. Dependences of relative elastic deflection values from the span length are proposed, which allows designing and calculating long-span structures using the simplified dynamic models. 


\section{References}

1. A.V. Benin, L.K. Dyachenko, V.N. Smirnov, Design and construction peculiarities of the 'Moscow-Kazan' high-speed railway mainline, Proceedings of St. Petersburg State Transport University (in Russian), 4, 45, 15-20 (2015)

2. L.K. Dyachenko, Dynamic calculations of HSR bridge superstructures for passenger trains running at the speed of up to $400 \mathrm{~km} / \mathrm{h}$, Proceedings of the International conference New technologies in bridge building from the past to the future (in Russian), St. Petersburg, 91-97 (2015)

3. V.N. Smirnov, A.A. Baranovsky, G.I. Bogdanov, D.E. Vorobyev, L.K. Dyachenko, V.V. Kondratov, Bridges on high-speed railways (St. Petersburg, in Russian, 2015)

4. Technical specifications: Artificial structures on Moscow-Kazan section of MoscowKazan-Ekaterinburg high-speed railway mainline. Design and construction norms and requirements, in Russian (St. Petersburg, 2014)

5. EN 1991-2 (2003): Eurocode 1: Actions on structures. P. 2: Traffic loads on bridges 